\title{
Factors Associated With Undernutrition Among Pregnant and Lactating Syrian Refugee Women in Jordan
}

\author{
Heyam F. Dalky, $\mathrm{PhD}, \mathrm{RN}^{1}$, Abeer Qandil, $\mathrm{PhD}, \mathrm{RN}^{1}$ \& Amani A. Alqwasmi, MSN, $\mathrm{RN}^{2}$ \\ ${ }^{1}$ Community Mental Health Department, Faculty of Nursing, Jordan University of Science and Technology, Irbid, \\ Jordan \\ ${ }^{2}$ Graduate Student, Community Mental Health Department, Faculty of Nursing, Jordan University of Science and \\ Technology, Irbid, Jordan \\ Correspondence: Heyam F. Dalky, Associate Professor, Faculty of Nursing, Jordan University of Science \& \\ Technology, P.O Box 3030, Irbid 22110, Jordan. Tel: 962-77-630-3092. E-mail: hfdalky@just.edu.jo
}

Received: January 21, 2018 Accepted: March 1, 2018 Online Published: March 5, 2018

doi:10.5539/gjhs.v10n4p58 URL: https://doi.org/10.5539/gjhs.v10n4p58

\begin{abstract}
Background: Maternal undernutrition is a public health issue and is reported to cause life-long and irreversible damage, with consequences at the individual, community, and national level. Many factors are reported to impact nutritional status for refugee pregnant or lactating women. Recently, Jordan has accepted an influx of refugees from Syria. Maternal undernutrition in pregnant and lactating Syrian women poses significant health risks.

Objective: To identify the relationship of undernutrition to underlying causes of socio-demographic, health and obstetric care, psychological wellbeing, social support, and marital violence among pregnant and lactating Syrian women attending obstetric outpatient clinics in Jordan.
\end{abstract}

Methods: The study was a cross-sectional assessment of 423 pregnant and lactating Syrian refugee women of established households within Jordan. Self-report questionnaires and anthropometric measurements were primary data sources.

Results: $49.2 \%(n=208)$ of participants were categorized as undernutrition (undernourished), a problem that is more prevailing among pregnant than lactating women. Statistical significance association was found for the variables extended family type, availability of health services, regular exercise, the trimester of pregnancy, low birth weight of the baby, and psychological well-being, when examined against undernutrition status.

Conclusion: Undernutrition is a significant health issue among women of reproductive age. This study is a building block for further research, yet it provides basic information on the effect of undernourishment on pregnant and lactating Syrian refugee women.

Keywords: lactating, pregnant, psychological wellbeing, social support, undernutrition

\section{Introduction}

Undernutrition is an important public health issue, particularly for vulnerable groups, including children and women of child-bearing age. Undernutrition has two major types; Protein-energy undernutrition or "Macro-nutrient deficiency" and "Micro-nutrient deficiency" (World Health Organization [WHO], 2012). Protein-energy undernutrition is the most serious form and occurs when the person consumes insufficient amount of calories from protein and other macronutrients; however, micronutrient deficiency in terms of mineral and vitamin deficiencies is also considered dangerous (WHO, 2012).

Malnutrition and undernutrition terms are being used interchangeably; however; malnutrition is a broader term that encompasses both over-nutrition in form of overweight and obesity and also undernutrition in the form of macro-nutrient and micro-nutrient deficiency. The term undernutrition is used for the purpose of this study, but the term malnutrition will also be encountered throughout this study as it was used by most of published studies to refer to undernutrition. Malnutrition in the form of over-nutrition is not the focus of this study.

According to a recent report of by McGuire, Food and Agricultural organization (FAO), International Fund for Agricultural Development (IFAD), and World Food Program (WFP), undernutrition is an important public health issue for women of child-bearing age and is especially relevant when resources are strained. Women who are 
pregnant or lactating are considered to be vulnerable for many reasons, including social and economic inequalities and special dietary requirements (McGuire, FAO, IFAD and WFP, 2015).

\subsection{The Case of Jordan}

Jordan is among the most financially disadvantaged countries in Middle-East, as denoted by limited natural resources and heavy reliance on foreign assistance (United Nations High Commissioner for Refugees [UNHCR], 2014). Based on data from 2010; 14.4 percent of the Jordanian population was found to be below the poverty line (UNHCR, 2014). However; this situation is expected to become more complex and severe in light of the dramatic changes through which the country is struggling as a result of economic crises and associated poverty as well as the refugee influx from surrounding countries (UNHCR, 2014).

Recently, the huge number of refugees coming from Syria after the Syrian civil war constituted the largest segment of the non-Jordanian part of the population living in Jordan (Department Of Statistics [DOS], 2016). Accordingly; about 1,265514 Syrian refugees are currently living in Jordan (DOS, 2016). The vast majority ( $84 \%$ ) of registered Syrian refugees live outside the country's main refugee camps of Zaatari and Azraq, in which two thirds are now living below the national poverty line (UNHCR, 2014).

The Jordanian health system is one of the best health systems in the region, especially in regards to maternal and child care. About $99 \%$ of Jordanian women received antenatal care from health care professionals, such as physicians, nurses or midwives (DOS, 2016). Antenatal care in Jordan is provided by trained health care professionals to ensure optimal pregnancy outcomes and to prevent complications. Routine antenatal care includes, but is not limited to, health education about proper diet during pregnancy and the need for special dietary concerns. It also includes general check-up for anemia and the prescription of supplements such as iron and folic acid (UNHCR, 2014). In regard to Syrian refugees; $82.2 \%$ of pregnant Syrian refugees received antenatal care with an average of 6.2 visits throughout their pregnancy UNHCR, 2014).

\subsection{Purpose of the Study}

The maternal mortality rate in 2013 in Jordan was reported at 50 per 100,000 live births, and for children under-five years of age; the mortality rate was 19 per 100,000 live births (WHO, 2015). Although these rates declined over years, it is still considered high. All types of malnutrition posses significant threats to human health. Maternal undernutrition, common in many developing countries; leads to poor fetal development, higher risk of pregnancy complications and thus, higher maternal mortality rates (WHO, 2015). Together, maternal and child undernutrition accounts for more than $10 \%$ of the global burden of disease (WHO, 2015).

Women, especially of child-bearing age, is considered to be among the most vulnerable groups for many reasons, such as social and economic inequalities and special dietary requirements (McGuire et al., 2015). Around half of pregnant women in developing countries are anemic and this contributed to $20 \%$ of all maternal deaths (WHO, 2012a). In addition; undernourished mothers are more likely to give birth to underweight babies, of which $20 \%$ die before age of five years (WHO, 2012a).

In Jordan; the issue of undernutrition among pregnant and lactating women (PLW) is not well understood. There is a lack of published research studies to cover the prevalence of undernutrition and its possible correlates, both in Jordanian citizen and non-citizen populations. To our current knowledge; there is no published study in Jordan that addresses the issue of undernutrition and its correlation in women who are pregnant or lactating. Although information from social media has described the poverty of Syrian refugees, specific information is lacking. Little is known about undernutrition of Syrian refugee women of chil-bearing age. To help fill this gap, this study was conducted.

The underlying premises of the present study were adapted from the United Nations Children's Emergency Fund (UNICEF) Conceptual framework for the Causes of Malnutrition, a model that demonstrates undernutrition has multiple causes. Whereas immediate causes are lack of food and healthcare, underlying causes include sociodemographic aspects, psychological wellbeing, and social support (UNICEF, 2016). It is known that inadequate food intake and medical care result in undernutrition. Therefore, the factors of research interest were factors that are likely to affect women of childbearing age. Based on the UNICEF model, the variables of interest of the current study were socio-economic demographics, health and obstetric issues, perceived social support, marital violence, and psychological wellbeing. The intent was to obtain a cross-sectional view of possible underlying causes toward providing needed healthcare and resources.

\section{Methods}

The study was designed as a cross-sectional, quantitative study wherein participating PLW were categorized as 
either in a state of undernutrition (undernourished) or non-undernutrition (nourished), as based on physical measurements taken at the time of the study. As per measures in Anthropometry Procedures Manual (Centers for Disease Control and Prevention, 2007), undernutrition was determined based on Body Mass Index (BMI), Middle Upper Arm Circumference (MUAC), and hemoglobin level.

\subsection{Sample and Setting}

The study was conducted in five nutrition clinics within primary health centers that are directed by one of non-governmental organizations located in urban areas of Middle and Northern Jordan. These five nutrition clinics are similar in the services they provide; each clinic has one nutritional officer or clinical nutrition nurse with background either in nursing or nutrition. The nutritional officer/nurse is responsible for assessing women and identifying those as moderate and severe acute malnutrition; especially for children under five years of age and those who are pregnant and lactating. These services are provided to all care users, especially Syrian refugees.

The sample consisted of Syrian PLW who were identified in the six clinics during the data collection period. The inclusion criteria required that the women be registered Syrian refugees with households within the country (outside of refugee camps), pregnant women in first, second or third trimester, or lactating mothers for infants below six months of age. The exclusion criteria related to Syrian pregnant women with abnormal pregnancies, such as molar and ectopic pregnancy, and lactating women for infants older than six months.

Non-probability convenience sampling was used to recruit eligible participants. All participants who met the eligibility criteria were recruited until the desired sample size was reached. Sample size was estimated by using the rule of 10 participants recruited for each variable or sub-variable. Thus, for the total number of the 40 variables; a sample size of 400 participants was needed at the minimum. Extra participants (23) were recruited to cover any possible attrition. The number of participants recruited from each of the five clinics was approximately the same, due to the similar number of PLW and cases of undernutrition at each clinic. Thus; a proportionate sampling was not needed.

\subsection{Study Instruments}

The study focused on the relationship of undernutrition vs. non-undernutrition in relation to six main research variables (demographics, health related issues, obstetric history psychological wellbeing, social support and marital violence). Primary data were physical measurements, demographic and general medical conditions, and self- reported responses on three screening instruments.

Using determinants taken at the time of sample recruitment, undernutrition was defined as BMI below 18.5, MUAC of $230 \mathrm{~mm}$ or less (Tang et al., 2013), and hemoglobin level below $11 \mathrm{~g} / \mathrm{dl}$. Hemoglobin test results were retrieved from PLW clinical records. Approximately half of the sample $(n=208)$ were designated as being undernourished.

The Mental Health Inventory (MHI-18, Arabic version) was used to measure general psychological distress and wellbeing (Veit \& Ware, 1983), the Multidimensional Scale of Perceived Social Support (MSPSS, Arabic version) was used to perceive type of support (Aroian, Templin, \& Ramaswamy, 2010), and the Abuse Assessment Screen (AAS, Arabic version) was used as an indicator of abuse (Calonge, 2004). The authors gave permission to use the instruments.

\subsection{Pilot Study}

A preliminary pilot study, using a convenience sample of 30 undernourished pregnant and lactating Jordanian women, was conducted to ensure clarity of questions of the Arabic version of the instruments. The results revealed instruments' psychometric prosperities are within acceptable ranges of reliability evidence.

\subsection{Ethical Consideration}

Approval for conducting this study was obtained from the Authors' host Academic Institute and from directors of the five clinics. Written consent was obtained from each participant. Anonymity was guaranteed, and potential participants were assured that their healthcare at the clinic did not depend upon their participation in the research.

\subsection{Data Collection and Analysis}

The data collection process spanned 15 weeks, with approximately three weeks spent at each clinic for data collection. Anthropometric measurements were collected before questionnaire completion to ensure data were available for PLW classification as undernutrition or non-undernutrition. The data were analysed using Statistical Package for the Social Science (SPSS), Version 22. Descriptive statistics (frequencies, percentages, mean, standard deviation) were used to describe the characteristics of the sample and variables of the study 
(demographics, health related issues, obstetric history psychological wellbeing, social support and marital violence). Inferential statistics using correlation tests were applied to identify relationship of the study variables.

\section{Results}

This study aimed to identify the relationship between variables (demographics, health related issues, obstetric history psychological wellbeing, social support and marital violence) and undernutrition among Syrian refugee pregnant and lactating women (PLW) who attend Obstetric outpatients clinics in Jordan.

\subsection{Characteristics of the Sample}

The number of participants approached and invited to participate was 439 Syrian PLW. Those who agreed to participate were 428 , giving an initial response rate of $97.49 \%$. Three participants were excluded because they did not meet the inclusion criteria; namely being not pregnant or lactating women. During data entry, no questionnaires were excluded because of missing data. Each questionnaire was checked for missing data, and those with missing data were returned to the participant to complete it if she agreed. The final total number of participants was 423 . The number of participants from each clinic were approximately equal; clinic one resulted in 85 , clinic two in 85 , clinic three in 88 , clinic four in 80 , and clinic five in 85 .

The mean age of the sample was $27.6(\mathrm{SD}=6.86)$, with $48.9 \%$ between $20-29$ years old. Most $(93 \%)$ were married and $64.5 \%$ belonged to nuclear families vs. extended families. Some $88.6 \%$ were of low income, and $62 \%$ reported availability of health services in their area. Of the total sample $(n=423), 70.9 \%(n=300)$ were pregnant, $29.1 \%(n$ $=123)$ were lactating, and $46.1 \%(\mathrm{n}=195)$ had high gravidity (greater than 4 births), as shown in Table 1 . Further, a majority $(85.8 \%, \mathrm{n}=363)$ reported they did not take prescribed medications, whereas $35.7 \%(\mathrm{n}=151)$ reported they took non-prescribed medication. Also, a majority $(81.3 \%, \mathrm{n}=344)$ reported they did not exercise regularly.

Table 1. Description of Demographic Characteristics of the Participants $(\mathrm{N}=423)$

\begin{tabular}{llcc}
\hline Demographics & & Frequency & Percentage (100\%) \\
\hline \multirow{2}{*}{ Age } & $<20$ & 49 & 11.6 \\
& $20-29$ & 207 & 48.9 \\
& $30-39$ & 148 & 35.0 \\
\multirow{2}{*}{ Employment } & $>40$ & 19 & 4.5 \\
\hline \multirow{3}{*}{ Income } & Yes & 38 & 9 \\
& No & 385 & 91 \\
\hline \multirow{3}{*}{ Marital status } & Poor Income & 325 & 86.2 \\
& Low Income & 50 & 13.3 \\
& Middle Income & 2 & 0.5 \\
\hline \multirow{2}{*}{ Family Type } & Married & 394 & 93.1 \\
& Divorced & 12 & 2.8 \\
\hline \multirow{2}{*}{ Exercise } & Separated & 9 & 2.1 \\
& Widow & 8 & 1.9 \\
\hline
\end{tabular}

Note. The table above reported results for some of the demographics due to space limitation.

In terms of nutrition, $49.2 \%(\mathrm{n}=208)$, were categorized as undernutrition (undernourished), with $50.8 \%(\mathrm{n}=215)$ categorized as non-undernutrition (nourished). Although half were considered to be undernourished, $5.4 \%(\mathrm{n}=23)$ had a BMI below 18.5, 14.4\% $(\mathrm{n}=61)$ had MUAC less than $230 \mathrm{~mm}$, whereas $40.4 \%$ had a Hemoglobin level below $11 \mathrm{~g} / \mathrm{dl}$. 


\subsection{Description of Obstetrics' Variables}

Assessment of the obstetric history revealed that the majority of women were pregnant $(\mathrm{n}=300,70.9 \%)$. However; women with high gravidity $(>=4)$ constituted about half of the study participants $(n=195,46.1 \%)$. About two third of women who participated in this study received antenatal care at least once $(\mathrm{n}=286,67.6)$, and the number of those who adhered to antenatal vitamins was high $(\mathrm{n}=341,80.6 \%)$. About two thirds of the study participants reported they were not suffering from any form of pregnancy complications $(n=328,77.5 \%)$. Also, about one third of them reported having aborted at least once during their life $(n=133,31.4 \%)$. The number of women who reported giving birth to a low birth weight baby are almost similar to those who reported having preterm deliveries; the numbers were, respectively, low birth weight $(\mathrm{n}=77,18.2 \%)$ and preterm delivery $(74,17.5 \%)$.

\subsection{Psychological Wellbeing among Study Participants}

Using the MHI-18 scale, the participants in the current study reported a mean total score of $52.31(\mathrm{SD}=14.59)$ with a range of (12.22 to 93.33). The subscales and total score ranges were from 0 to 100 , with higher scores indicating better mental health. The mean total scores of the four subscales of the mental health inventory (MHI) are illustrated in Table 2.

Table 2. Description of Mental Health Inventory Score $(\mathrm{N}=423)$

\begin{tabular}{lcc}
\hline Psychological well being & Mean & SD \\
\hline Total MHI & 52.31 & 14.59 \\
Anxiety & 50.56 & 18.88 \\
Depression & 50.87 & 24.90 \\
Behavioral control & 58.32 & 17.66 \\
Positive Affect & 48.16 & 22.63 \\
\hline
\end{tabular}

*MHI=Mental Health Inventory.

The Mental Health inventory has no cut off points; therefore, for statistical purposes, MHI raw scores were categorized into percentiles. Below $25^{\text {th }}$ percentile was considered as low psychological wellbeing, between $25^{\text {th }}$ and $75^{\text {th }}$ was considered as average, and above $75^{\text {th }}$ was considered better psychological well-being. Categorized scores of the subscales are shown in Table 3.

Table 3. Description of the Categorized MHI Scores ( $\mathrm{N}=423$ )

\begin{tabular}{llcc}
\hline Psychological wellbeing & & Frequency & Percentage \% \\
\hline \multirow{3}{*}{ Total score } & Low & 37 & 8.7 \\
& Average & 318 & 75.2 \\
& Better & 68 & 16.1 \\
\hline \multirow{3}{*}{ Anxiety } & Low & 89 & 21.0 \\
& Average & 232 & 54.8 \\
& Better & 102 & 24.1 \\
\hline \multirow{3}{*}{ Depression } & Low & 108 & 25.5 \\
& Average & 177 & 41.8 \\
& Better & 138 & 32.6 \\
\hline \multirow{3}{*}{ Behavioural control } & Low & 28 & 6.6 \\
& Average & 270 & 63.8 \\
& Better & 125 & 29.6 \\
\hline \multirow{3}{*}{ Positive Affect } & Low & 121 & 28.6 \\
& Average & 213 & 50.4 \\
& Better & 89 & 21.0 \\
\hline
\end{tabular}




\subsection{Perceived Social Support of the Participants}

The MSPSS mean total score was $4.8(\mathrm{SD}=1.38)$, which is approximately mid-point on a 1 to 7 scale. This mean is considered medium, according to a three cut-off-point categorization by Aroian and colleagues (2010). In this case, low is $<4$, medium is $4-<6$, and high is 6-7. However, the Arabic version of MSPSS does not consider these cut off points. Instead, the closer the score is to the highest point (7) is considered the highest component for social support. In addition, results in this study showed the major source of social support was almost equal among the husband $(\mathrm{M}=5.4, \mathrm{SD}=1.80)$, family $(\mathrm{M}=4.67, \mathrm{SD}=1.79)$, or friends $(\mathrm{M}=4.24, \mathrm{SD}=1.95)$.

\subsection{Marital Violence Among Participants}

One third of the study sample reported being abused by their spouses in the last year of their life $(n=149,35.2 \%)$. The report of marital abuse was similar among both undernutrition $17.7 \%(\mathrm{n}=75)$, and non-undernutrition $(17.4 \%$, $\mathrm{n}=74$ ) groups.

\subsection{Relationship between Study Variables and Undernutrition}

To obtain these results; chi square was used to test associations between demographics, health issues, obstetrics, psychological wellbeing, perceived social support and marital violence. The main variable was the nutritional status of pregnant and lactating women who participated in the study, namely, being undernourished or non-undernourished.

In examining the relationship between participants' demographics and nutritional status, a statistical significance difference between undernourished versus non-undernourished PLW was found in regards to family type; namely nuclear and extended $\left(\chi^{2}(1, \mathrm{n}=423)=5.223, \mathrm{p}=.022\right)$. Results showed that more than half of PLW who belong to extended families $(n=85,56.7 \%)$ suffer from undernutrition. In addition; when the nutritional status was compared in relation to regular exercise, a statistical significance difference was found $\left(\chi^{2}(1, n=423)=8.74, p=.003\right)$. The results showed that more than half of those who did not report the performance of regular exercise suffered from undernutrition $(\mathrm{n}=181,52.6 \%)$. Other demographics were not found to be statistically associated with nutritional status.

After performing chi square of independence to test the relationship between obstetrics and nutritional status, a significant association was found between pregnant or lactating and having undernutrition $\left(\chi^{2}(1, \mathrm{n}=423)=8.337\right.$, $\mathrm{p}=.004)$. Results showed that undernutrition was more prevalent among pregnant women $(\mathrm{n}=161,53.7 \%)$ than among lactating $(n=47,38.2 \%)$. In addition, in examining the relationship between nutritional status and specific trimester of pregnancy, a significant association was observed $\left(\chi^{2}(3, n=423)=12.990, p=.005\right)$. Results showed that the lowest percentage of undernutrition was among pregnant women in their first trimester $(n=14,37.8 \%)$ and the highest was among those in their third trimester $(\mathrm{n}=92,57.2 \%)$. Another statistically significant relationship was revealed between birth weight of the baby and nutritional status $\left(\chi^{2}(3, n=423)=9.364, p=.025\right)$. The results showed that PLW who are undernourished tend to give birth to a low birth weight baby.

In regard to psychological well-being, a significant statistical relationship was found between psychological wellbeing when examined against nutritional status $\left(\chi^{2}(2, n=423)=7.836, p=.020\right)$. Nearly, one third $(n=23$, $33.8 \%$ ) of those who were above the $75^{\text {th }}$ percentile (better psychological well-being) were undernourished. None of the subscales showed a significant relationship when examined against nutritional status. Finally, there was no statistical significance relationship between perceived social support and its subscales and violence variables when examined against nutritional status.

\section{Discussion}

This study aimed to identify the relationship between variables (demographics, health related issues, obstetric history psychological wellbeing, social support and marital violence) and undernutrition among Syrian pregnant and lactating women who attended outpatient obstetric clinics in Jordan.

The majority of study participants were not employed (91\%), and had a low income of "living on less than two dollars per family member", a result that was anticipated. In the Syrian culture, as in most Arab cultures, the woman's major responsibility is to care for children and the house; it is the man's responsibility to work and provide the family with needed money and life expenses. However, this figure is changing due to the difficult life challenges and the revolution of gender equality (World Bank Report, 2013). Indeed, most cultures are witnessing more contributions and collaborations between men and women in order to assure a respectable life for themselves and their families. Another possible reason behind low employment among pregnant or lactating women relates to work laws and politics. Many Syrian refugees could find it difficult to obtain work permission from the hosting country. Further, illegal employment may subject the refugees to legal or law violations. 
In this study, results showed that pregnant and lactating women who live in extended families are more likely to be undernourished than those who live in nuclear families. This may be explained by the family income and food being distributed to a large number of family members who already live in poverty and shortage of resources. In addition; women are considered to be among the vulnerable population groups that are subject to social inequalities. Also, women give more attention to feeding the children and husband and subsequently, are the last person to eat or eats smaller portion of foods (Shannon, Mahmud, Asfia, \& Ali, 2008).

In regards to lifestyle habits, more than half of those who did not report performance of regular exercise suffered from undernutrition. One explanation for no exercise performance is that women who are undernourished could have general weakness and fatigue, which might contribute to their inability to exercise regularly. The multi-task role of most Syrian women in this study limits the available time for extra activities, including regular exercise. Educational programs to increase attention and awareness about the importance of regular exercise for PLW are essential.

In this study, women who are pregnant were more likely to be undernourished than those who are lactating. In addition, those in the third trimester were more likely to be undernourished than those in their first and second trimester. This result is consistent with studies of the association between gravidity and undernutrition among pregnant and lactating women (Davies et al., 2012; Haileslassie, Mulugeta \& Girma, 2013). However, another study contradicted this finding (Al-Farsi et al., 2011). Women in the Syrian culture, as well as most Arab cultures, are the caretaker of children, husband and the home, and having many children limits the mothers' ability to take care of themselves.

The number of PLW who reported giving birth to a low birth weight baby (18.2\%) is similar to those who reported having preterm deliveries $(17.5 \%)$. However, PLW in the study who were undernourished tended to give birth to low birth weight babies. In one study, positive effects were found between protein-energy supplementation on birth weight and still birth in relation to maternal weight (Imdad \& Bhutta, 2012). In a systemic review, it was found that preconception and peri-conception intake of vitamins and minerals is associated with reduced risk for low birth weight, small for gestational age, and preterm delivery (Ramakrishnan, Grant, Goldenberg, Zongrone, \& Martorell, 2012).

A significant statistical relationship was found for general psychological wellbeing, although not he subscales, when examined against nutritional status. Women in the later trimester tended to be undernourished. Although, in this study, nutritional status failed to have significant association with subscales of the general psychological wellbeing scale, a possible explanation for psychological wellbeing affect on nutritional status is through its effect on appetite, as was found by a study that targeted older people (Engel et al., 2011). However, in a study that was conducted in India among pregnant women, depression was not found to be associated with nutritional status (Lukose et al., 2014).

An explanation for nutritional status associated with general psychological wellbeing failed to associate with subscales of anxiety, depression, behavioural control and positive affect could be inherent in the unique characteristics of each subscale. Each subscale has different characteristics and effects on the human beings that differ from one person to another. For example; it is well known that persons react differently to depression. For example, some tend to overeat while others refuse to eat.

In the present study, the mean total score of social support using MSPSS was 4.82 of a 7-point scale. The score may be over reported, as in Arab cultures, women are supported by the men of the family, both extended and nuclear. As refugees, paternal and male family support may be fractured, due to death, disease, hardships, and accidents. The PLW of the current study indicated support was received almost equally from husband, family and friends. It is not unusual for refugee women to seek support from female friends as they congregate at marketplaces, schools, clinics, and places of worship. Traditionally, wives look to their husbands for support, and the husband's socioeconomic status influences undernutrition in wives (Ghosh, 2014).

Lastly, about one third of pregnant and lactating women PLW who participated in this study reported being abused by their spouses in the last year of their lives. However, there was no statistical significance in the relationship between marital abuse and nutritional status. Cultural and personal factors (fear, shame, insecurity) are possible factors to consider as they may prohibit public disclosure of abuse. However, a study by Hernandez and colleagues (2014) reported an association between intimate partner violence and food insecurity, a state which could lead to undernutrition. Further, studies have found that physical and sexual violence from an intimate partner increased the risk of chronic undernutrition in women of reproductive age (Dougé, Lehman, \& McCall-Hosenfeld, 2014; Rahman, Nakamura, Seino, Kizuki, 2013). Hence, results are to be considered with caution when studying sensitive or critical variables, such as marital abuse, physical violence, food insecurities, or the like. 


\subsection{Strength and Limitations}

This is the first known study in Jordan that addressed the issue of undernutrition in Syrian PLW refugees and its relationship to variables of demographics, health and obstetric issues, psychological wellbeing, social support, and marital violence. However, the study was a cross-sectional assessment. Further, the sample itself was not comprehensive of Syrian refugees as the sample was drawn from women who presented at urban clinics. Also; the number of PLW may be underestimated as it is not known how many registered PLW Syrian refugees did not seek medical attention. In addition, respondents' replies may be biased as cultural influences may have caused more positive responses than warranted.

\section{Conclusion}

The study provided unique information about undernutrition in pregnant women in Jordan after the Syrian crises and the influx of refugees. This study aimed to qualify the relationship between possibly contributing variables and undernutrition among pregnant and lactating refugee Syrian women established in households within Jordan. Study results indicated that nurses can play a significant role in identifying undernourished PLW using a triage approach of physical measurements (e.g. MUAC, BMI and hemoglobin level). Clinic and community resources can be used to supplement diet. Because undernutrition appears to be more prevalent in pregnant than lactating women, they are most likely to require healthcare attention and food as well as educational resources. Also, study results suggested a relationship between mental health and nutrition. Pregnant refugee women with better psychological wellbeing were found to be at less risk for undernutrition.

\section{Acknowledgements}

The study was funded by the Deanship of Research at Jordan University of Science \& Technology, Research Grant No: 20150349.

\section{Author Contributions}

Heyam Dalky (H.D.) devised the project, the main conceptual ideas and proof outline. H.D. and Abeer Qandil (A.Q.) worked out almost all of the technical details. H.D., A.Q. and Amani ALqawasmi (A.A.) were involved in planning and drafted the manuscript. A.A. collected and processed the data. A.Q. and A.A. performed the measurements and the analysis. H.D. aided in interpreting the results and worked on the manuscript.

\section{Competing Interests Statement}

The authors declare that they have no competing or potential conflicts of interest.

\section{References}

Al-Farsi, Y. M., Brooks, D. R., Werler, M. M., Cabral, H. J., Al-Shafei, M. A., \& Wallenburg, H. C. (2011). Effect of high parity on occurrence of anemia in pregnancy: A cohort study. BMC Pregnancy and Childbirth, 11, 7-15. https://doi.org/10.1186/1471-2393-11-7

Aroian, K., Templin, T. N., \& Ramaswamy, V. (2010). Health care for Women International Adaptation and Psychometric Evaluation of the Multidimensional Scale of Perceived Social Support for Arab immigrant women. Peace and Conflict. Retrieved from https://doi.org/10.1080/07399330903052145

Calonge, N. (2004). Screening for family and intimate partner violence: Recommendation statement. American Family Physician, 70(4), 747-751. https://doi.org/10.1370/afm.128

Centers for Disease Control and Prevention. (2007). Anthropometry Procedures Manual - National Health and Nutrition Examination Survey. Retrieved from https://www.cdc.gov/nchs/data/nhanes/nhanes 07_08/manual_an.pdf

Davies, H. R., Visser, J., Tomlinson, M., Rotherham-Borus, M. J., LeRoux, I., \& Gissane, C. (2012). An investigation into the influence of socioeconomic variables on gestational Body Mass Index in pregnant women living in a peri-urban settlement, South Africa. Maternal and Child Health Journal, 16(8), 1732-1741. https://doi.org/10.1007/s10995-011-0869-7

Dougé, N., Lehman, E. B., \& McCall-Hosenfeld, J. S. (2014). Social support and employment status modify the effect of intimate partner violence on depression symptom severity in women: Results from the 2006 Behavioral Risk Factor Surveillance System Survey. Women's Health Issues, 24(4), e425-e434. https://doi.org/10.1016/j.whi.2014.03.006

Department Of Statistics. (2016). Population and housing census. Department of Statistics. http://doi.org/10.1017/CBO9781107415324.004 
Engel, J. H., Siewerdt, F., Jackson, R., Akobundu, U., Wait, C., \& Sahyoun, N. (2011). Hardiness, depression, and emotional well-being and their association with appetite in older adults. Journal of the American Geriatrics Society, 59(3), 482-487. https://doi.org/10.1111/j.1532-5415.2010.03274.x

Ghosh, S. (2014). Exploring vulnerability of undernutrition among wives: Do husbands' attributes matter? Journal of Health Management, 16(3), 423-439. https://doi.org/10.1177/0972063414539616

Haileslassie, K., Mulugeta, A., \& Girma, M. (2013). Feeding practices, nutritional status and associated factors of lactating women in Samre Woreda, South Eastern Zone of Tigray, Ethiopia. Nutrition Journal, 1, 12-28. https://doi.org/10.1186/1475-2891-12-28

Hernandez, D. C., Marshall, A., \& Mineo, C. (2014). Maternal depression mediates the association between intimate partner violence and food insecurity. Journal of Women's Health (2002), 23(1), 29-37. https://doi.org/doi.org/10.1089/jwh.2012.4224

Imdad, A., \& Bhutta, Z. A. (2012). Maternal nutrition and birth outcomes: Effect of balanced protein-energy supplementation. Paediatric and Perinatal Epidemiology, 26(SUPPL. 1), 178-190. https://doi.org/10.1111/j.1365-3016.2012.01308.x

Kochhar, R. (2015). A global middle class is more promise than reality: From 2001 to 2011, nearly 700 million step out of poverty, but most only barely. Pew Research Center. Retrieved from http://www.pewglobal.org/files/2015/07/Global-Middle-Class-Report_FINAL_7-8-15.pdf

Lukose, A., Ramthal, A., Thomas, T., Bosch, R., Kurpad, A. V., Duggan, C., \& Srinivasan, K. (2014). Nutritional factors associated with antenatal depressive symptoms in the early stage of pregnancy among urban South Indian Women. Maternal and Child Health Journal, 18(1), 161-170. https://doi.org/10.1007/s10995-013-1249-2

McGuire, Sh., FAO, IFAD, \& WFP. (2015). The State of Food Insecurity in the World 2015: Meeting of the 2015 International Hunger Targets: Taking Stock of Uneven Progress. Rome: FAO, 2015. Advances in Nutrition, 6(5), 623-624. https://doi.org/10.3945/an.115.009936

Rahman, M., Nakamura, K., Seino, K., \& Kizuki, M. (2013). Intimate partner violence and chronic undernutrition among married Bangladeshi women of reproductive age: Are the poor uniquely disadvantaged? European Journal of Clinical Nutrition, 67(3), 301-307. https://doi.org/10.1038/ejen.2012.202

Ramakrishnan, U., Grant, F., Goldenberg, T., Zongrone, A., \& Martorell, R. (2012). Effect of women's nutrition before and during early pregnancy on maternal and infant outcomes: A systematic review. Paediatric and Perinatal Epidemiology, 26, 285-301. https://doi.org/10.1111/j.1365-3016.2012.01281.x

Shannon, K., Mahmud, Z., Asfia, A., \& Ali, M. (2008). The social and environmental factors underlying maternal malnutrition in rural Bangladesh: implications for reproductive health and nutrition programs. Health Care for Women International, 29(8), 826-840. https://doi.org/10.1080/07399330802269493

Tang, A., Dong, K., Deitchler, M., Chung, M., Maalouf-Manasseh, A., Tumilowicz, C., \& Wanke, C. (2013). Use of cutoffs for mid-upper arm circumference (MUAC) as an indicator or predictor of nutritional and healthrelated outcomes in adolescents and adults: A systematic review. Retrieved from https://www.fantaproject.org/sites/default/files/resources/MUAC\%20Systematic\%20Review\%20_Nov\%20 19.pdf

Talwar, P., Kumaraswamy, N., \& Ar, M. F. (2013). Perceived Social Support, Stress and Gender Differences among University Students : A Cross Sectional Study. Malaysian Journal of Psychiatry, 22(2), 42-49.

UNHCR. (2014). Syria Regional response plan 6, UNHCR. Retreived from http://www.unhcr.org/syriarrp6/docs/Syria-rrp6-full-report.pdf

UNICEF. (2016). The State of the World's Children. Retrieved from https://www.unicef.org/sowc98/fig5.htm

United Nations Population Fund (UNFPA). (2015). Women and Girls in the Syria Crisis: UNFPA response. Retrieved from https://www.unfpa.org/sites/default/files/resource-pdf/UNFPA-FACTSANDFIGURES5[4].pdf

Veit, C. T., \& Ware. J. E. Jr. (1983). The structure of psychological distress and well-being in general populations. Journal of Consulting Clinical Psychology, 51(5), 730-42. https://doi.org/10.1037/0022-006X.51.5.730

World Bank Report. (2013). Opening doors: gender equality and development in the Middle East and North Africa: Main report (English). MENA development report. Washington DC: World Bank. Retrieved from 
http://documents.worldbank.org/curated/en/338381468279877854/Main-report

WHO. (2015). Syrian Refugee Health Access Survey in Jordan. John Hopkins University. Retrieved from https://reliefweb.int/report/jordan/syrian-refugee-health-access-survey-jordan-december-2014

\section{Copyrights}

Copyright for this article is retained by the author(s), with first publication rights granted to the journal.

This is an open-access article distributed under the terms and conditions of the Creative Commons Attribution license (http://creativecommons.org/licenses/by/4.0/). 